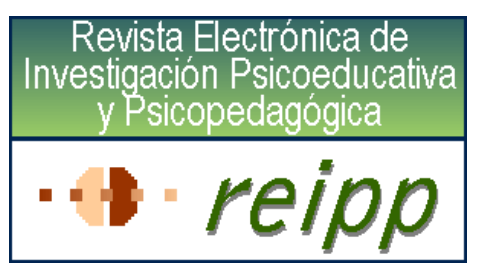

REVISTA ELECTRÓNICA DE

INVESTIGACIÓN PSICOEDUCATIVA Y PSICOPEDAGÓGICA

No 1 (2) 2003. ISSN: 1696-2095

\title{
Sensibilidad de algunas pruebas estandarizadas para evaluar el funcionamiento de la atención ejecutiva en niños de siete años
}

Luis J. Fuentes*, Carmen González**, Angeles F. Estévez*, José A. Carranza**, Mayte Daza*, María D. Galián**, y Dolores Alvarez*

- Universidad de Almería / ** Universidad de Murcia

España

1fuentes@ual.es 


\section{RESUMEN}

Introducción. La utilización de pruebas estandarizadas para la evaluación de las habilidades atencionales, es una práctica habitual dentro de contextos clínicos y educativos. Puesto que el concepto de atención resulta algo ambiguo, este estudio intenta examinar la relación entre algunas de estas pruebas y la atención ejecutiva, tal y como es conceptualizada en las aproximaciones más recientes de la neurociencia cognitiva.

Método. Se administraron algunos subtests del WISC-R, la parte A y B del Trial Making Tests y la versión de tarjetas de la tarea Stroop a un grupo de niños de siete años. A continuación los niños llevaron a cabo una versión computerizada de la tarea Stroop, la cual, según algunos estudios de neuroimagen, estaría asociada con el circuito neural implicado en la atención ejecutiva.

Resultados. Los niños puntuaron diferencialmente únicamente en los subtests de Figuras Incompletas, en Dígitos y en la parte B del Trial Making Test, produciendo diferentes patrones de interferencia en el efecto Stroop. Además, las puntuaciones obtenidas en ambas versiones de la tarea Stroop no correlacionaron.

Discusión. Los resultados parecen indicar que estas pruebas son sensibles al funcionamiento de la atención ejecutiva en niños de edad escolar. Por otra parte, la ausencia de correlación entre las dos versiones de la tarea Stroop sugiere que en la versión de tarjetas los mecanismos atencionales podrían ser diferentes a los implicados en la versión informatizada.

Palabras clave: Tests estandarizados, WISC-R, Trial Making Test, tarea Stroop, atención ejecutiva, asesoramiento psicopedagógico, evaluación neuropsicológica 


\section{Introducción}

Ciertos tests estandarizados, como la escala de inteligencia de Weschler para niños (WISC del inglés Weschler Intelligence Scales for Children), se han convertido en poderosos instrumentos, ampliamente utilizados en contextos clínicos y educativos para evaluar funciones cognitivas en niños de edad escolar. Los autores han considerado que la atención, junto con otras operaciones cognitivas, juega un papel importante en la ejecución de estos tests. Sin embargo, aunque todavía hoy día el término atención resulta algo ambiguo, desde recientes aproximaciones neurocognitivas, la atención es considerada como un fenómeno multicomponencial que ejerce funciones de control a través de la coordinación de diferentes redes. Cada una de estas redes llevaría a cabo funciones atencionales específicas (Fuentes, en prensa). Algunas de estas funciones estarían relacionadas con la orientación de la atención a localizaciones espaciales anticipando la aparición de estímulos relevantes. Desde un punto de vista funcional, esta red de orientación operaría cuando la atención es cambiada hacia una localización en la que aparece una señal que indica el lugar en el que tendrá lugar un estímulo. Las operaciones de desenganche, movimiento y enganche involucradas en este tipo de atención, dependen del lóbulo parietal posterior, los colículos superiores y el núcleo pulvinar del tálamo, respectivamente (para una revisión, ver Posner y Petersen, 1990; Posner y Raichle, 1994). Esta red es de suma importancia, ya que nos ayuda en la búsqueda de estímulos potencialmente relevantes, favoreciendo así su procesamiento de alto nivel. Por otra parte, se cree que las funciones cognitivas relacionadas con el procesamiento de más alto nivel estarían bajo el control de otro sistema atencional superior conocido como red atencional ejecutiva.

La atención ejecutiva se ha asociado con la activacion de áreas anteriores del cerebro, concretamente, porciones del lóbulo prefrontal dorsolateral y áreas frontales, incluído el cortex cingulado anterior y el área motora suplementaria. Estas áreas se activan en situaciones que requieren planificación, toma de decisiones, corrección de errores, ejecución de respuestas que no están bien aprendidas o contrarrestar respuestas habituales (o automáticas) (Posner y DiGirolamo, 1998), todas ellas actividades que requieren control ejecutivo.

El objetivo de este estudio fue evaluar si las escalas estandarizadas que supuestamente miden atención, como las utilizadas en contextos neuropsicológicos y psicopedagógicos, se relacionan con la red ejecutiva del sistema atencional en niños de edad escolar. Las pruebas elegidas fueron las Figuras incompletas, Historietas, Dígitos y Rompecabezas de la Escala de 
Inteligencia de Wechler para niños Revisada (el WISC-R; Weschler , 1974), la versión de tarjetas de la tarea Stroop y el Trial Making Test (la parte A y B). También se utilizó una versión computerizada de la tarea Stroop como medida de atención ejecutiva.

\section{La Escala de Inteligencia de Weschler para niños - Revisada (WISC-R)}

El WISC-R es considerado como un instrumento para medir la inteligencia general, y como una herramienta de diagnóstico para detectar déficit cognitivos en contextos neuropsicológicos, psicoeducativos y clínicos. Consta de doce subtests, 6 de ellos conforman la Escala verbal y los 6 restantes la Escala Manipulativa. A continuación describiremos brevemente algunos de los subtests que han sido considerados como medidas de atención.

En el subtest de Figuras Incompletas se pide a los participantes que descubran y nombren (o indiquen) la parte omitida de un dibujo incompleto. Las operaciones cognitivas involucradas incluyen detección y búsqueda visual; recuerdo de items en la memoria visual; comparación entre estímulos y recuerdo visual de imágenes; y lo que es más importante, la habilidad del sujeto para seleccionar los detalles esenciales de los dibujos.

En el subtest de Historietas el participante tiene que ordenar una serie de tarjetas con dibujos de forma que, los dibujos que hay en ellas cuenten una pequeña historia con sentido. Esta tarea mide la habilidad del sujeto para comprender la idea general de la historia y poder realizarla con éxito. Las operaciones cognitivas involucradas incluyen habilidad para la planificación, anticipación de consecuencias de situaciones iniciales, organización visual y secuenciación temporal.

La prueba de Dígitos requiere que el niño repita una serie de dígitos que el experimentador presenta auditivamente. Esta prueba mide memoria auditiva a corto plazo y atención. Tiene dos partes: Dígitos en orden Directo y Dígitos en orden Inverso. Puesto que las dos partes parecen implicar diferentes componentes de la memoria a corto plazo (working memory), la mayoría de los autores señalan que ambas pruebas deben analizarse de forma separada (Lezak, 1995). En el test de Dígitos Directos los niños tienen que repetir series que van desde dos hasta nueve dígitos en el mismo orden que son presentados por el examinador. Esta prueba requiere principalmente almacenamiento a corto plazo de información auditiva en la working memory. En el test de Dígitos Inversos, los niños deben repetir series que van desde dos hasta ocho dígitos, en orden inverso (o contrario) al presentado por el examinador. Esta prue- 
ba implica la manipulación activa de la información almacenada (v.g., el sistema de control ejecutivo de la working memory; Baddeley, 1986).

En el subtest de Laberintos el niño tiene que resolver laberintos de papel y lápiz que difieren en nivel de dificultad. En cada laberinto el examinando tiene que dibujar una línea desde el centro hasta el exterior, sin cruzar ninguna de las líneas que representan las paredes del laberinto. Este test requiere atención a las direcciones y parece medir la habilidad del niño para la planificación y la organización perceptual.

\section{Trial Making Tests (TMT)}

El TMT fue desarrollado por la Armada estadounidense en 1944. Consta de dos partes. En el subtest A (TMT-A) el sujeto tiene que unir círculos colocados aleatoriamente con números (1-25) en orden numérico, tan rápido como le sea posible. En el subtest B (TMT-B), la tarea consiste en conectar alternativamente círculos con números (1-13) y con letras (A-L), en sus respectivas secuencias lo más rápido posible. Investigaciones previas con pacientes neurológicos han mostrado que una ejecución deficiente en el TMT-B podría estar relacionada con un fallo en el procesamiento inhibitorio (Amieva et al., 1998). El procesamiento inhibitorio ha sido considerado como una de las funciones importantes de la red atencional ejecutiva (Fuentes, en prensa; Fuentes, Vivas, \& Humphreys, 1999; Posner y Raichle, 1994).

\section{La Tarea Stroop}

La tarea Stroop (Stroop, 1935) ha sido ampliamente utilizada para medir la atención selectiva tanto en estudios neuropsicológicos como cognitivos. En esta tarea se pide a los participantes que nombren el color en el que parecen pintadas las palabras. Para ello, los participantes tienen que atender a la dimensión relevante de la palabra (el color de la tinta) al mismo tiempo que ignoran la dimensión irrelevante pero también la más predominante (el significado de la palabra). Cuando los participantes tienen que nombrar el color de palabras incongruentes (v.g., la palabra ROJO escrita en color azul), tienen dificultades para ignorar los efectos intrusivos del significado de las palabras, mostrando un deterioro en la ejecución (mayor tiempo de reacción y/o porcentaje de errores) en comparación con una condición neutral en la que se utilizan estímulos sin sentido (v.g., un conjunto de Xs). De esta forma, el efecto de interferencia Stroop se obtiene comparando la ejecución de los participantes en la condición incongruente con la obtenida en la condición neutral. 
En la versión computerizada (de ensayo simple) de la tarea Stroop, los estímulos son presentados individualmente en el centro de la pantalla del ordenador. Estudios con técnicas de neuroimagen (v.g., tomografía por emisión de positrones, -PET-; resonancia magnética funcional, fMRI), han revelado que el efecto de interferencia Stroop se asocia con la activación en el córtex cingulado anterior (Fan, Flombaum, McCandliss, Thomas, y Posner, 2003; Pardo, Pardo, Janer y Raicle, 1990) o en el córtex prefrontal (Taylor, Kornblum, Lauber, Minoshima y Koeppe, 1997), áreas cerebrales que han sido relacionadas con la atención ejecutiva (para una revisión, ver Bush, Luu y Posner, 2000). La interferencia Stroop únicamente puede observarse a partir de los 6-7 años de edad, coincidiendo con la necesaria habilidad de lectura que hace que el procesamiento semántico sea un proceso automático, y con la maduración del córtex frontal y prefrontal involucrados en el control ejecutivo del procesamiento de la información.

Existen otras versiones de la tarea original empleada por Stroop en 1935. En la versión de tarjetas, todos los estímulos Stroop se presentan simultáneamente en una tarjeta. La primera tarjeta contiene una lista de palabras escritas en color negro. Los participantes tienen que leer las palabras. La interferencia Stroop se calcula a partir de las otras dos tarjetas en las que los participantes tienen que nombrar los estímulos de color. Una de las tarjetas contiene filas de Xs en diferentes colores y sirven como condición neutral. La otra tarjeta contiene palabras de color incongruentes y sirve como condición incongruente. Puesto que la versión computerizada ha mostrado reflejar las operaciones de la atención ejecutiva, y la versión de tarjetas se utiliza más frecuentemente en estudios neuropsicológicos y psicopedagógicos, una comparación entre las dos formas de interferencia Stroop es especialmente relevante para determinar si la versión de tarjetas de la tarea Stroop resulta realmente sensible al control ejecutivo.

Si la versión de tarjetas mide atención ejecutiva, deberíamos encontrar una correlación con las puntuaciones obtenidas en la versión computerizada, la cual ha demostrado ser útil para medir atención ejecutiva.

Los subtests del WISC-R, la parte A y B del TMT y la versión de tarjetas de la tarea Stroop, fueron administrados a niños de siete años. A continuación, debían realizar la versión computerizada de la tarea Stroop para medir el funcionamiento de su atención ejecutiva. El objetivo de este estudio fue investigar si los niños que puntuaran diferencialmente en estos tests mostrarían patrones diferentes de interferencia Stroop, y por lo tanto en el funcionamien- 
to de las operaciones de la atención ejecutiva. Esto nos posibilitará seleccionar los subtests relacionados con la atención ejecutiva cuando sean utilizados en el marco clínico, psicoeducativo y psicopedagógico.

\section{Método.}

\section{Participantes}

Participaron doscientos cuatro niños de los colegios españones Francisco de Goya (Almería), Lope de Vega (Almería) y San Buenaventura (Murcia). Todos ellos tenían 7 años y se encontraban cursando el segundo grado de la escuela primaria. Ninguno de los niños manifestó tener problemas con los colores. En el momento de la prueba todos tenían visión normal o corregida. Ninguno de ellos mostraba problemas de lectura o aprendizaje.

\section{Instrumentos y aparatos}

Para los tests estadarizados, a todos los niños se les administraron cuatros subtest del WISC-R: Figuras Incompletas, Historietas, Dígitos (Directos e Inversos) y Laberintos. Los niños también completaron la parte A y B del Trial Making Test y la versión de tarjetas de la tarea Stroop (Golden, 1978; test distribuido por ediciones TEA).

Para medir la atención ejecutiva utilizamos una versión computerizada de la tarea Stroop. En esta tarea, los estímulos objetivo eran las palabras ROJO, AZUL y VERDE y una fila de cuatro Xs que podían aparecer en color rojo, azul o verde. Los estímulos Stoop eran presentados uno a uno en la pantalla a color (VGA) de un ordenador compatible con IBM. Las respuestas de naming fueron registradas ensayo a ensayo a través de una llave vocal conectada con el puerto paralelo del ordenador.

\section{Procedimiento}

Para la tarea Stroop computerizada, el ordenador mostraba un signo mas $(+)$ durante $500 \mathrm{~ms}$ que servía como punto de fijación. A continuación se presentaba el estímulo objetivo o target que permanecía en pantalla hasta la respuesta de los participantes. Estos tenían que nombrar el color del target y el experimentador introducía un código para que más tarde se pudieran codificar las medidas de precisión. Después de una demora de 2000 ms, comenzaba un nuevo ensayo con la presentación del punto de fijación. Los participantes realizaron un bloque de 72 ensayos. Los 12 primeros eran ensayos de práctica que no se incluyeron en los 
análisis. Los 60 restantes eran ensayos experimentales, 30 para la condición neutral (10 ensayos por cada color), y 30 para la condición incongruente (10 por cada color). Las variables dependientes fueron los tiempos de reacción (TRs) y el porcentaje de errores. La interferencia Stroop se midió restando los TRs y el porcentaje de errores de la condición incongruente menos los de la condición neutral.

Todos los tests estandarizados (WISC, TMT y las tarjetas Stroop) fueron administrados de acuerdo con el procedimiento estandar utilizado en contextos clínicos y psicoeducativos.

\section{Análisis de datos}

Para Figuras Incompletas, Historietas, Dígitos y Laberintos, los niños fueron divididos en dos grupos de acuerdo con la puntuación media. Los niños que puntuaron por debajo o por encima de la media fueron categorizados como bajos o altos en cada test, respectivamente (ver González, Fuentes, Carranza y Estévez, 2001, para un método de análisis similar). Para el TMT, las puntuaciones fueron 0 si los niños eran incapaces de realizar la tarea, 1 si la completaban pero necesitaban más tiempo del establecido como tiempo límite ( 2 minutos y 30 segundos), y 2 si completaban la tarea dentro del tiempo límite. Los niños con una puntuación de 0 fueron clasificados como bajos y aquellos que obtuvieron una puntuación de 2 fueron clasificados como altos para los propósitos del análisis.

También realizamos análisis de correlación para evaluar la relación entre las dos versiones de la tarea Stroop. Se obtuvo una puntuación de interferencia Stroop (incongruente neutral) de las dos tareas para calcular la correlación.

Para la tarea Stroop computerizada se realizó un ANOVA factorial mixto 2 x 2 x 2 , con el género (chicos y chicas) y las puntuaciones del test (bajos vs. altos) como factores entre-grupos, y la condición Stroop (neutral vs. incongruente) como factor intrasujetos.

\section{Resultados}

\section{Efecto Stroop General}

La Tabla 1 muestra la media de las medianas de los TRs y el porcentaje de errores para la tarea Stroop computerizada. El efecto principal de la condición fue significativo tanto 
para los TRs, $\mathrm{F}(1,202)=300.49, \mathrm{p}<0.0001$; como para los errores, $\mathrm{F}(1,202)=68.73 ; \mathrm{p}<$ 0.0001. Los estímulos incongruentes produjeron TRs más largos y más errores que los estímulos neutrales. Es decir, observamos el efecto típico de interferencia Stroop. Ni la variable género ni la interacción Género x Condición fueron significativas. El análisis de correlación de Pearson mostró una baja correlación entre ambas medidas del efecto Stroop $(\mathrm{r}=0.18$, para los TRs; y r $=0.012$, para los errores).

Tabla 1. Media de Medianas de los Tiempos de Reacción y Porcentaje de Errores para la versión Computerizada de la Tarea Stroop.

\begin{tabular}{lccccc}
\hline & \multicolumn{2}{c}{ Chicos } & & \multicolumn{2}{c}{ Chicas } \\
\cline { 5 - 6 } Condición & TR & PE & TR & PE \\
\hline Neutral & 944 & 3.8 & 962 & 3.0 \\
Incongruente & 1123 & 7.7 & 1173 & 6.9 \\
\hline
\end{tabular}

Nota $. \mathrm{TR}=$ tiempo de reacción; $\mathrm{PE}=$ porcentaje de errores.

Interacciones entre los tests estandarizados y el efecto de interferencia Stroop

Los tiempos de reacción y el porcentaje de errores de la versión computerizada de la tarea Stroop actuaron como variables dependientes en los siguientes análisis. Dado que en estos análisis el género no interactuó con ningún otro factor, los datos de esta variable fueron agrupados. Con objeto de simplificar, las puntuaciones altas o bajas de los niños en los subtest sólo se presentan en aquellos casos en los que se encontró una interacción Efecto Stroop x Test significativa (ver Figura 1).

Para el test de Figuras Incompletas, el análisis del porcentaje de errores mostró un efectos principal significativo de la puntuación y de la condición, $\mathrm{F}(1,184)=9.99, \mathrm{p}<0.01 ; \mathrm{y}$ $\mathrm{F}(1,184)=62.84, \mathrm{p}<0.001$, respectivamente. Los niños que puntuaron bajo tuvieron más errores que los niños que puntuaron alto (6.4 vs. 4.3\%), y la condición incongruente produjo más errores que la condición neutral (7.2 vs. 3.4\%). Y lo que es más importante, la interacción Puntuación x Condición también fue significativa, $F(1,184)=3.78, p=0.05$. El análisis 
de esta interacción indicaba que los niños que habían puntuado bajo tuvieron mayor interferencia Stroop que los niños que puntuaron alto (ver Figura 1a).

Para el test de Dígitos Inversos, el análisis de los TRs mostró un efecto principal significativo tanto de la puntuación como de la condición, $\mathrm{F}(1,202)=13.20, \mathrm{p}<0.01$; y $\mathrm{F}(1,202)$ $=310.60, \mathrm{p}<0.0001$, respectivamente. Los niños que puntuaron bajo tuvieron mayores TRs que los que puntuaron alto (1108 vs. $1005 \mathrm{~ms}$ ), y la condición incongruente produjo TRs mayores que la condición neutral (1156 vs. $958 \mathrm{msec}$ ). La interacción Puntuación x Condición también fue significativa $F(1,202)=5.89, p<0.025$. Nuevamente, los niños que puntuaron bajo en este test mostraron una interferencia Stroop mayor que la obtenida por los niños que puntuaron alto (ver Figura 1b).

Para el TMT-B, el análisis del porcentaje de errores mostró efectos principales significativos de la puntuación y de la condición, $\mathrm{F}(1,142)=7.21, \mathrm{p}<0.01 ; \mathrm{y} F(1,142)=33.08$, $\mathrm{p}<$ 0.001, respectivamente. Los niños que puntuaron bajo cometieron más errores que los niños que puntuaron alto (6.0 vs. 3.9\%), y la condición incongruente produjo el doble de errores que la condición neutral (6.6 vs. 3.3\%). Al igual que anteriormente, la interacción Puntuación x Condición fue significativa, $\mathrm{F}(1,142)=4.12, \mathrm{p}<0.05$. Los niños que puntuaron bajo en el TMT-B mostraron una mayor interferencia Stroop que los niños que puntuaron alto (ver Figura $1 \mathrm{c})$.

Figura 1. Efecto Stroop en la versión computerizada en función de la puntuación en Figuras Incompletas (a), Dígitos Inversos (b) y en el Trial Making Test B (c).

a)

Figuras Incompletas

Neutral $\square$ Incongr.

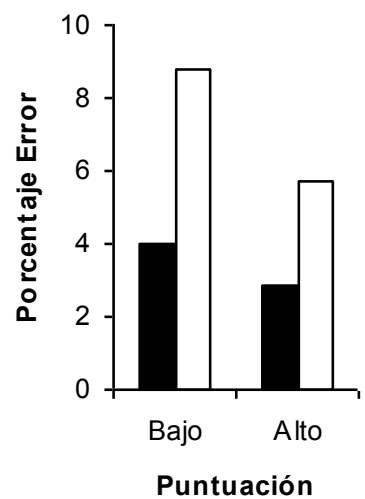

b)

Dígitos Inversos

- Neutral $\square$ Incongr.

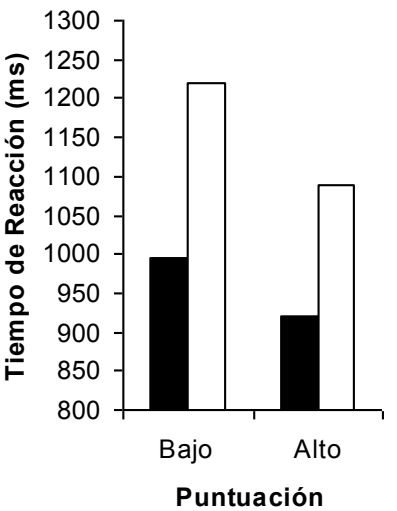

c)

Test Trail Making (B)

- Neutral $\square$ Incongr.

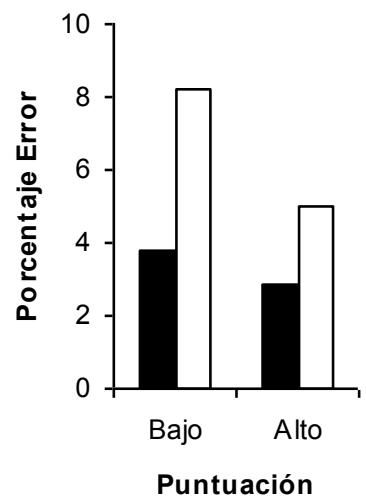


Con ninguno de los demás tests se observó efectos Stroop diferenciales en función de la puntuación, ni con los TRs ni con los errores.

\section{Discusión}

El objetivo del presente trabajo era evaluar la relación entre algunos tests estandarizados considerados como medidas de atención y la atención ejecutiva medida a través de una versión computerizada de la tarea Stroop. Existen varias razones de peso para estudiar tal relación. En primer lugar, pruebas como el WISC-R, el TMT y la versión de tarjetas de la tarea Stroop son ámpliamente utilizadas en contextos educativos y neuropsicologicos proporcionando una medida de las capacidades atencionales. En segundo lugar el concepto de atención, donde ésta ha comenzado a ser considerada como un sistema formado por diferentes redes atencionales, cada una de ellas con distintas funciones. (Fuentes, en prensa; Posner y Raichle, 1994). Por tanto, parece que existe cierta disociación entre cómo se concibe la atención en el contexto clínico/educativo y cómo se define en los estudios neuro-cognitivos. En el presente estudio nos preguntamos si algunos de los tests que se cree miden atención en niños de edad escolar se relacionan con la atención ejecutiva.

Un análisis preliminar muestra que tanto los chicos como las chicas son suceptibles a la interferencia que producen las palabras de color cuando realizan la tarea Stroop computerizada. Estos resultados confirman los obtenidos en estudios previos en los que se ha visto que niños de 7 años muestran efectos de interferencia Stroop (Bonino y Ciairano, 1997; González et al., 2001; Schiller, 1966). Sin embargo, cuando nos centramos en las interacciones entre los tests estandarizados y la interferencia Stroop, los resultados mostraron que sólo 3 de los tests produjeron diferencias en el efecto Stroop.

Cuando se utiliza una tarea Stroop, que los estudios de neuroimagen han asociado claramente con la red ejecutiva, sólo Figuras Incompletas (errores), Dígitos Inversos (TRs) y el TMT-B (errores) parecen ser sensibles a una interferencia Stroop diferencial. Y lo que es más importante, las puntuaciones de ambas versiones de la tarea Stroop no correlacionaron.

A partir de estos resultados podemos perfilar dos importantes implicaciones. En primer lugar, únicamente dos subtests de la escala WISC-R que supuestamente miden atención (Figuras Incompletas y Dígitos Inversos), y la parte B del Trial Making Test estaban re- 
lacionados con la atención ejecutiva en niños de 7 años cuando los efectos de interferencia son medidos a través de la tarea Stroop computerizada. La pobre ejecución que se observa en estos test podría ser debida a una disfunción del circuito neural asociado con la red atencional ejecutiva.

En segundo lugar, dados estos resultados, la versión de tarjetas de la tarea Stroop puede no ser adecuada como un índice de atención ejecutiva, al menos en esta edad. Esto estaría apoyado, aunque indirectamente, por la pobre correlación entre las dos versiones de la tarea Stroop.

Estudios previos han observado estas disociaciones entre las dos versiones cuando se mide la atención en pacientes esquizofrénicos (Boucart, Mobarek, Cuervo, \& Danion, 1999). Boucart et al. (1999) han señalado que existen importantes diferencias metodológicas entre las dos versiones. Mientras que en la versión computerizada los estímulos son presentado uno a uno en la pantalla, en la versión de tarjetas todos los estímulos se presentan simultáneamente, teniendo el sujeto que seleccionar el target actual a través de un gran número de distractores. Además, en la versión computerizada las condiciones están mezcladas pero en la versión de tarjetas están bloqueadas. Todas estas diferencias podrían sugerir que en cada versión de la tarea predominan diferentes mecanismos atencionales. Mientras que la resolución de conflicto, junto con la red atencional ejecutva, podría ser predominante en la versión computerizada (Botvinick, Nystrom, Fissell, Carter y Cohen, 1999), la localización del target y el filtraje de información distractora, función en la que el núcleo pulvinar del tálamo juega un papel importante (LaBerge y Buchsbaum, 1990), podría predominar en la versión de tarjetas (Boucart et al., 1999), estando implicada la red atencional de orientación.

Investigaciones futuras deberán determinar si las relaciones encontradas en el presente estudio pueden ser establecidas entre otros tests estandarizados y formas de atención, y en función del desarrollo.

\section{Nota de los autores}

Esta investigación fue subvencionada por las becas BSO2003-00930 y PB98-0379 del Ministerio de Ciencia y Tecnología. Los autores desean dar las gracias a los niños y niñas por su participación y al equipo humano de los colegios Lope de Vega (Almería), Francisco de Goya (Almería) y San Buenaventura (Murcia) por su inestimable ayuda.

Luis J. Fuentes. 


\section{Referencias}

Amieva, H., Lafont, S., Auriacombe, S., Rainville, C., Orgogozo, J.M., Dartigues, J.F., \& Fabrigoule, C. (1998). Analysis of error types in the Trial Making Test evidences an inhibitory deficit in dementia of the Alzheimer Type. Journal of Clinical and Experimental Neuropsychology, 20, 280-285.

Baddeley, A. (1986). Working Memory. Oxford, England: Oxford University Press.

Bonino, S., \& Ciairano, S. (1997). Effetto Stroop e capacità di inhibizione: Una ricerca in campo evolutivo. [The Stroop Effect and inhibition capacity: Research in the developmental field.] Giornale Italiano di Psicologia, 24, 587-603.

Botvinick, M., Nystrom, L.E., Fissell, K., Carter, C.S., \& Cohen, J.D. (1999). Conflict monitoring versus selection-for-action in anterior cingulate cortex. Nature, 402, 179-181.

Boucart, M., Mobarek, N., Cuervo, C., \& Danion, J.M. (1999). What is the nature of increased interference in schizophrenia? Acta Psychologica, 101, 3-25.

Bush, G., Luu, P., \& Posner, M.I. (2000). Cognitive and emotional influences in anterior cingulate cortex. Trends in Cognitive Sciences, 4, 215-222.

Fan, J., Flombaum, J.I., McCandliss, B.D., Thomas, K.M., \& Posner, M.I. (2003). Cognitive and brain mechanisms of conflict. NeuroImage, 18, 42-57.

Fuentes, L.J. (en prensa). Inhibitory processing in the attentional networks. In M.I. Posner (Ed.), Attention. New York: Guilford Press.

Fuentes, L.J., Vivas, A.B. \& Humphreys, G.W. (1999). Inhibitory mechanisms of attentional networks: Spatial and semantic inhibitory processing. Journal of Experimental Psychology: Human Perception and Performance, 25, 1114-1126.

Golden, C.J. (1978). Stroop color and word test. Chicago: Stoelting.

González, C., Fuentes, L.J., Carranza, J.A., \& Estévez, A.F. (2001). Temperament and attention in the self-regulation of seven-year-old children. Personality and Individual Differences, 30, 931-946.

LaBerge, D., \& Buchsbaum, M.S. (1990). Positron emission tomographic measurements of pulvinar activity during an attention task. Journal of Neuroscience, 10, 613-619.

Lezak, M.D. (1995). Neuropsychological assessment. New York: Oxford University Press.

Pardo, J.V., Pardo, P.J., Janer, K.W., \& Raichle, M E. (1990). The anterior cingulate cortex mediates processing selection in the Stroop attentional conflict paradigm. Proceedings of the National Academy of Science, 87, 256-259. 
Posner, M.I., \& DiGirolamo, G. J. (1998). Conflict, target detection and cognitive control. In R. Parasuraman (Ed.), The attentive brain (pp. 401-423). Cambridge, M. A.: MIT Press.

Posner, M.I. \& Petersen, S.E. (1990). The attention system of the human brain. Annual Review of Neuroscience, 13, 25-42.

Posner, M.I., \& Raichle, M E. (1994). Images of mind. New York: Scientific American Library.

Schiller, P.H. (1966). Developmental study of color-word interference. Journal of Experimental Psychology, 72, 105-108.

Stroop, J R. (1935). Studies of interference in serial verbal reaction. Journal of Experimental Psychology, 18, 643-662.

Taylor, S.F., Kornblum, S., Lauber, E.J., Minoshima, S., \& Koeppe, R.A. (1997). Isolation of specific interference processing in the Stroop task: PET activation studies. NeuroImage, 6, 81-92.

Weschler, D. (1974). Manual for the Weschler Intelligence Scale for Children-Revised. San Antonio, TX: The Psychological Corporation. 